\title{
Soliton Resonances, Black Holes and Madelung Fluid
}

\author{
Oktay K PASHAEV ${ }^{\dagger_{1}, \dagger_{2}}$ and Jyh-Hao LEE ${ }^{\ddagger}$ \\ †1 Joint Institute for Nuclear Research, Dubna (Moscow), 141980, Russian Federation \\ E-mail: pashaev@vxjinr.jinr.ru \\ $\dagger_{2}$ Department of Mathematics, Izmir Institute of Technology Cankaya-Izmir, Turkey \\ E-mail: pashaev@likya.iyte.edu.tr \\ $¥$ Institute of Mathematics, Academia Sinica, Taipei, 11529, Taiwan \\ E-mail: leejh@ccvax.sinica.edu.tw
}

\begin{abstract}
The reaction-diffusion system realizing a particular gauge fixing condition of the Jackiw-Teitelboim gravity is represented as a coupled pair of Burgers equations with positive and negative viscosity. For acoustic metric in the Madelung fluid representation the space-time points where dispersion change the sign correspond to the event horizon, while shock soliton solutions to the black holes, creating under collision the resonance states.
\end{abstract}

\section{Introduction}

A connection between black holes physics and hydrodynamics established by Unruh [1] has been applied recently to simulate the Hawking radiation and other quantum effects related to event horizon and ergoregions in supersonic fluid flows and in a superfluid [2]. Recently an implication appeared that the black-hole-like phenomena might take place in the nonlinear Schrödinger type equation, where black holes of a constant curvature spacetime have been related to the soliton-like solutions for dissipative version of the NLS in the form of the Reaction-Diffusion system (RD) [3]. These solutions called dissipatons, characterize completely the black hole horizon, the Hawking temperature and the causal structure [4]. Here we present a simple two fluid interpretation of black holes in terms of Madelung fluid and describe a collision of black holes showing a novel character creating resonance states with a specific lifetime.

\section{Envelope solitons and quantum potential}

We begin with a problem of the NLS soliton subject to the influence of the so called "quantum potential" and described by the equation

$$
i \partial_{t} \psi+\partial_{x}^{2} \psi+\frac{\Lambda}{4}|\psi|^{2} \psi=s \frac{\partial_{x}^{2}|\psi|}{|\psi|} \psi
$$


where the r.h.s. represents contribution from the quantum potential. The potential was introduced by L de Broglie and has been explored by D Bohm to make a hidden-variable theory in quantum mechanics. In a more recent context it was considered in the stochastic mechanics as producing non-classical diffusion connected with several problems. The linear Schrödinger equation with quantum potential was considered by Auberson and Sabatier [5]. Below we consider the self-consistent potential $U=-\frac{\Lambda}{4}|\psi|^{2}$. Properties of Eq. (1) drastically depend on the value $s$. Decomposing the wave function $\psi=e^{R-i S}$ we have a system describing the so called Madelung fluid: the continuity equation for density $\rho=|\psi|^{2}=e^{2 R}$ and the Hamilton-Jacobi equation for the velocity potential $V(x, t)=-2 \partial_{x} S$, which after taking gradient is hydrodynamic equation

$$
\partial_{t} \rho+\partial_{x}(\rho V)=0, \quad \frac{\partial V}{\partial t}+V \frac{\partial V}{\partial x}+\frac{\partial}{\partial x} 2\left[U+(s-1) U_{Q}\right]=0 .
$$

The second equation along with nonlinear potential $U$, includes an extra contribution corresponding to the presence of quantum potential $U_{Q}$. Then, a particular particle acceleration given by the total derivative of $V$ would obey the Newton's equation of motion explaining why $U_{Q}$ is called quantum potential.

\section{Madelung fluid and Reaction-Diffusion system}

First we consider the case $s<1$. Rescaling the time and the phase $t=(1-s)^{-\frac{1}{2}} \tilde{t}$, $S(x, t)=(1-s)^{\frac{1}{2}} \tilde{S}(x, \tilde{t})$, in terms of new complex function $\tilde{\psi}=e^{R-i \tilde{S}}$ we get the usual NLS equation with renormalized coupling constant $\Lambda /(1-s)$. But situation changes drastically if $s>1$ and the system can not be simplified in terms of one complex function. Thus rescaling the time and the phase $t=(s-1)^{-\frac{1}{2}} \tilde{t}, S(x, t)=(s-1)^{\frac{1}{2}} \tilde{S}(x, \tilde{t})$, we introduce two new real functions (in what follows we skip the tilde sign), $e^{(+)}=\exp (R+S)$, $e^{(-)}=-\exp (R-S)$, and obtain the Reaction-Diffusion (RD) system

$$
\pm \partial_{t} e^{( \pm)}=\partial_{x}^{2} e^{( \pm)}+\frac{\Lambda}{4} e^{(+)} e^{(-)} e^{( \pm)} .
$$

It is worth to note that unusual negative value for diffusion coefficient in the second equation is crucial for existence of the Hamiltonian structure and integrability of the model. For $\Lambda<0$ it admits dissipaton solution

$$
e^{( \pm)}= \pm\left(\frac{8}{-\Lambda}\right)^{\frac{1}{2}} k e^{ \pm\left[\left(\frac{1}{4} v^{2}+k^{2}\right) t-\frac{1}{2} v x\right]} \cosh ^{-1}\left[k\left(x-v t-x_{0}\right)\right],
$$

with exponentially growing and decaying components, but with perfect solitonic shape for $O(1,1)$ scalar product $e^{(+)} e^{(-)}$.

\section{Gravitational interpretation}

Defining two dimensional metric tensor in terms of the Einstein-Cartan orthonormal set of basis vector fields $g_{\mu \nu}=e_{\mu}^{a} e_{\nu}^{b} \eta_{a b}=\frac{1}{2}\left(e_{\mu}^{(+)} e_{\nu}^{(-)}+e_{\nu}^{(+)} e_{\mu}^{(-)}\right)$, where $e_{\mu}^{( \pm)}=e_{\mu}^{0} \pm e_{\mu}^{1}=$ $\left(e_{0}^{( \pm)}, e_{1}^{( \pm)}\right), \eta_{a b}=\operatorname{diag}(-1,1)$, one can formulates the $(1+1)$-dimensiomal gravity model 
as the so called BF gauge field theory with $S O(2,1)$ Poincaré gauge group [3, 4]. Fixing gauge freedom by conditions $e_{0}^{( \pm)}= \pm \partial_{x} e^{( \pm)}, e_{1}^{( \pm)} \equiv e^{( \pm)}$such that

$$
g_{00}=-\frac{\partial e^{(+)}}{\partial x} \frac{\partial e^{(-)}}{\partial x}, \quad g_{11}=e^{(+)} e^{(-)}, \quad g_{01}=\frac{1}{2}\left(\frac{\partial e^{(+)}}{\partial x} e^{(-)}-e^{(+)} \frac{\partial e^{(-)}}{\partial x}\right)
$$

we find that $e^{( \pm)}$satisfy (3) when the metric describes two dimensional pseudo-Riemannian space-time with a constant curvature $\Lambda: R=g^{\mu \nu} R_{\mu \nu}=\Lambda$ (the Jackiw-Teitelboim gravity). It allow us to establish a correspondence between geometrical and physical characteristics of the model so that $g_{00}$ component is the dispersive part of energy density, while $g_{11}$ and $g_{01}$ the mass and momentum densities correspondingly. Calculating the mass, momentum and the energy

$$
E=2 \int_{-\infty}^{\infty}\left[\partial_{x} e^{(+)} \partial_{x} e^{(-)}-\frac{\Lambda}{8}\left(e^{(+)} e^{(-)}\right)^{2}\right] d x
$$

for the one-dissipaton $(4),(\Lambda<0)$,

$$
M=\frac{16}{-\Lambda}|k|, \quad P=M v, \quad E=\frac{M v^{2}}{2}+\frac{\Lambda^{2}}{384} M^{3} .
$$

we have non-relativistic quasi-particle of non-negative mass $M$ and momentum $P$, with the positive rest energy $E_{0}=E(v=0)=\frac{\Lambda^{2}}{384} M^{3}$.

\section{Resonance dispersion and event horizon}

Creation and annihilation processes for these quasi-particles lead to the resonance states. The decay of a dissipaton in the rest on the pair of dissipatons is allowed only if the rest energy is positive. Since the rest energy satisfies inequality

$$
E_{0}=\frac{\Lambda^{2}}{384} M^{3}=\frac{\Lambda^{2}}{384}\left(M_{1}+M_{2}\right)^{3}>\frac{\Lambda^{2}}{384}\left(M_{1}^{3}+M_{2}^{3}\right)=E_{0(1)}+E_{0(2)},
$$

such that $\Delta E_{0}=E_{0}-\left(E_{0(1)}+E_{0(2)}\right)>0$, it allows two dissipatons creation. Actually the resonance states existence relates to the form of dispersive part of energy density (6) which is nonpositive definite and has geometrical meaning of the metric tensor component $\epsilon_{0}=-2 g_{00}$. Then at the space-time points $\left(x_{H}, t_{H}\right)$, where dispersion and $g_{00}$ changes the sign we have the event horizon.

\section{Hydrodynamical representation}

For vanishing curvature $\Lambda=0$, the RD system (3) reduces to the pair of diffusion and anti-diffusion equations. By the Cole-Hopf transformation it can be represented as the decoupled pair of Burgers equations. This suggests to introduce for $\Lambda \neq 0$ the pair of velocity fields $u^{+}=-2\left(e_{x}^{(+)} / e^{(+)}\right), u^{-}=+2\left(e_{x}^{(-)} / e^{(-)}\right)$, such that instead of $\mathrm{RD}(3)$ we have the coupled system of Burgers equations with positive and negative viscosity and potential function $U=-\frac{\Lambda}{4} \rho$

$$
u_{t}^{ \pm}+u^{ \pm} u_{x}^{ \pm}= \pm u_{x x}^{ \pm}-2 U_{x}, \quad \frac{U_{x}}{U}=-\frac{1}{2}\left(u^{+}-u^{-}\right) .
$$



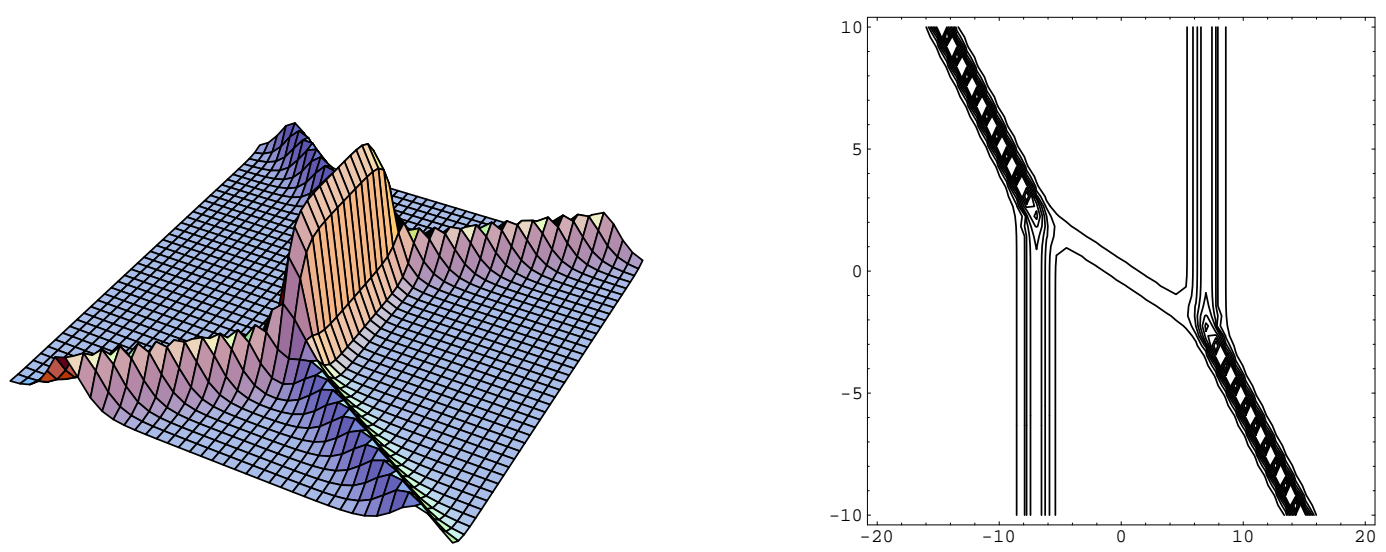

Figure 1. a) 3D plot of dissipatons resonance collision; b) Contour plot of exchange-type collision.

These relative velocities $u^{ \pm}=V \mp V_{Q}$ characterize two motions, the center of mass motion with $V \equiv-2 \partial_{x} S$ and internal oscillations in the envelope with velocity $V_{Q} \equiv \frac{\partial_{x} \rho}{\rho}$. The last one is the "quantum velocity" describing stochastic diffusion generated by quantum potential. In this hydrodynamical representation the metric tensor (5) acquires form

$$
g_{00}=\frac{1}{4} \rho\left(V_{Q}^{2}-V^{2}\right)=-\frac{1}{4} \rho u^{+} u^{-}, \quad g_{11}=-\rho, \quad g_{01}=\frac{1}{2} \rho V=\frac{1}{4} \rho\left(u^{+}+u^{-}\right),
$$

which is similar to the Arnowitt-Deser-Misner split of a $(1+1)$-dimensional Lorentzian space-time corresponding to the so-called acoustic metric [2], derived by Unruh for the sound waves in a fluid [1]. So the event horizon defined by $g_{00}=0$ appears at points with vanishing velocity $u^{+}=0$ or $u^{-}=0$ (or $V= \pm V_{Q}$ ). To one-dissipaton (4) corresponds the pair of shock solitons of (8)

$$
u^{ \pm}=v \pm 2 k \tanh k(x-v t)
$$

and the event horizon, appearing only for $|v| \leq 2|k|$, is defined by $\tanh k(x-v t)= \pm v / 2 k$.

If function $2 V /\left(V_{Q}^{2}-V^{2}\right)$ is integrable we sinchronize the space-time by introducing new time $d \tau=d t-2 V /\left(V_{Q}^{2}-V^{2}\right) d \xi$ and obtain Schwarzschild type black hole metric

$$
d s^{2}=\rho\left[\frac{1}{4}\left(V_{Q}^{2}-V^{2}\right)(d \tau)^{2}-\frac{V_{Q}^{2}}{V_{Q}^{2}-V^{2}}(d \xi)^{2}\right] .
$$

\section{Soliton resonances}

The bilinear representation for RD system (3) is

$$
\left( \pm D_{t}-D_{x}^{2}\right)\left(G^{ \pm} \circ F\right)=0, \quad D_{x}^{2}(F \circ F)=-2 G^{+} G^{-}
$$

where $e^{( \pm)}=(-8 / \Lambda)^{\frac{1}{2}} G^{ \pm} / F$, and the product $e^{(+)} e^{(-)}=-\frac{8}{-\Lambda} \partial_{x}^{2}(\log F)$. 
The one-dissipaton is given by $G^{ \pm}= \pm e^{\eta_{1}^{ \pm}}, F=1+e^{\eta_{1}^{+}+\eta_{1}^{-}+\phi_{1,1}}, e^{\phi_{1,1}}=\left(k_{1}^{+}+k_{1}^{-}\right)^{-2}$, and in terms $k \equiv\left(k_{1}^{+}+k_{1}^{-}\right) / 2, v \equiv-\left(k_{1}^{+}-k_{1}^{-}\right)$it acquires the form (4). For twodissipaton solution we have

$$
F=1+\sum_{i, j=1}^{2} \frac{e^{\eta_{i}^{+}+\eta_{j}^{-}}}{\left(k_{i j}^{+-}\right)^{2}}+\left(\frac{\breve{k}_{12}^{++} \breve{k}_{12}^{--}}{k_{12}^{+-} k_{21}^{+-} k_{11}^{+-} k_{22}^{+-}}\right)^{2} e^{\eta_{1}^{+}+\eta_{1}^{-}+\eta_{2}^{+}+\eta_{2}^{-}}
$$

where $k_{i j}^{a b} \equiv k_{i}^{a}+k_{j}^{b}, \breve{k}_{i j}^{a b} \equiv k_{i}^{a}-k_{j}^{b}, \eta_{i}^{ \pm} \equiv k_{i}^{ \pm} x \pm\left(k_{i}^{ \pm}\right)^{2} t+\eta^{ \pm(0)}$.

In general case it describes collision of two different dissipatons with amplitudes $k_{12}^{+-} / 2$ and $k_{21}^{+-} / 2$ and velocities $v_{12}=-\breve{k}_{12}^{+-}$and $v_{21}=-\breve{k}_{21}^{+-}$correspondingly. Depending of $k_{i}^{a}$ and positions shift the interaction might be the resonance-type (Fig. 1a.) and exchangetype (Fig. 1b.).

\section{References}

[1] Unruh W G, Phys. Rev. Lett., 1981, V.46, 1351.

[2] Visser M, Class. Quant. Grav., 1998, V.15, 1767;

Jacobson T A and Volovik G E, Phys. Rev. D, 1998, V.58, 064021.

[3] Martina L, Pashaev O K and Soliani G, Class. Quant. Grav., 1997, V.14, 3179;

Pashaev O K, Nucl. Phys. B, 1997, V.57, 338.

[4] Martina L, Pashaev O K and Soliani G, Phys. Rev. D, 1998, V.58, 084025.

[5] Auberson G and Sabatier P C, J. Math. Phys., 1994, V.35, 4028. 